\title{
A Heterogeneous Animated Platform for Educational Participatory Simulations
}

\author{
Bill Tomlinson \\ ACE (Arts Computation Engineering) program \\ University of California, Irvine \\ wmt@uci.edu
}

\begin{abstract}
This paper describes a multi-user interactive installation featuring real time animated creatures and a mobile interaction paradigm. This paradigm has been designed to serve as a platform for education in a variety of content domains. Drawing on previous research in mobile computing and animated educational systems, this project contributes a novel metaphor for interactions among real and virtual creatures and worlds. This "Land/Water" metaphor offers that virtual space is like land for virtual creatures, and real space is like water for them. The interaction paradigm involves groups of animated creatures that live on desktop screens, and may be transported from screen to screen by means of mobile Tablet PCs carried by human participants. This paper presents a working implementation of this paradigm and describes its use as an educational tool.
\end{abstract}

Keywords: Human-Computer Interaction, Handheld/Mobile Devices, Virtual/3D Environments, Participatory Simulations

\section{INTRODUCTION}

The continual development of new computational technologies presents the opportunity for the creation of novel modes of interaction that may contribute to educational processes. Computer graphics have been embraced by the educational community in a wide variety of domains such as chemistry (Wilson, 2002) or history (Jenkins, 2002). More recently, mobile computational devices have begun to play a role in education through participatory simulations, e.g., (Klopfer \& Woodruff, 2003), and university campus deployments, e.g., (Tatar et al., 2003). This paper presents an interaction paradigm that embraces both computer graphics and mobile devices in an educational context and describes a working implementation of this paradigm. This interaction paradigm and implementations based on it offer a viable platform for presenting a range of educational content areas in a compelling interactive way.

The interaction paradigm presented here is a multi-user experience involving several fixed computer displays and several mobile handheld devices (see Figure 1). Each fixed display serves as a "virtual island" inhabited by populations of 3D animated virtual creatures. When a human participant brings one of the mobile devices (a "virtual raft") close to the screen, one of the creatures on the virtual island moves onto the virtual raft. The participant may then carry that creature to a different virtual island; once the raft is close enough to the other island, the creature can move off the handheld onto the other desktop screen. This interaction paradigm, where people participate in the migration and dispersal of individuals and populations, encourages an active engagement from participants in the unfolding of the natural or cultural history existing on the islands. This interaction may serve as a platform for a range of educational content, including anthropology, social science, mythology, ecology and environmental biology (such as the study of biodiversity or invasive species.) For a better sense of the interaction, please view the following short video:

http://www. ics .uci.edu/ wmt/movies/HomeschoolVideo.mov

The core metaphor in this interaction paradigm draws a parallel between the land/water distinction in the real world and the virtual space/real space distinction. In this "Land/Water" metaphor, virtual space serves as land for virtual creatures, and real space functions as water. Virtual creatures may move around in virtual space, but are 
not able to move around in real space; similarly, real land animals are able to operate effectively on land but not nearly so well in water.

This metaphor provides a set of organizing principles for an interactive educational simulation. It distinguishes the operational domain of virtual creatures from that of people, thereby providing a way of explaining how people are meant to interact with the system. It provides inspiration for subject matter that may be effectively conveyed through this kind of simulation. And it provides guidance for the visual, acoustic, tactile and other elements of the interactive experience. While the platform is not ideal for all topics of education, it does provide a useful framework for exploring topics that deal with groups of people (e.g., social or cultural education, see Figure 2), groups of biological organisms (ecology, evolution, etc.) and potentially other topics (math, languages) as well.

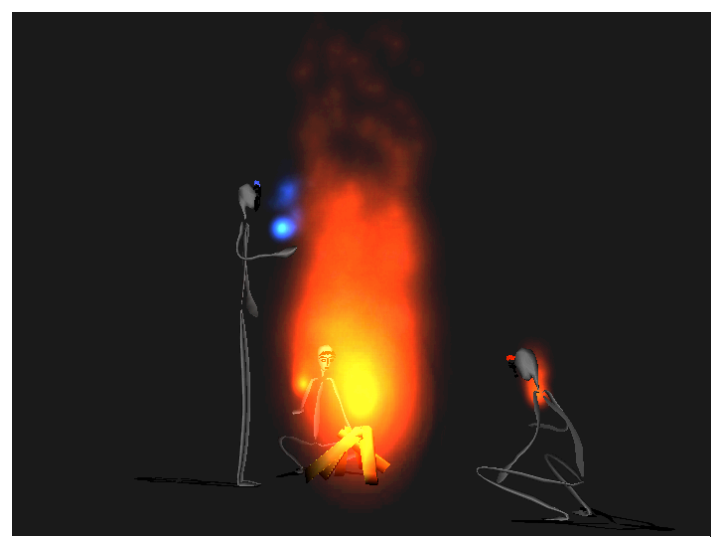

Figure 2: A community of autonomous animated characters inhabits each virtual island in one version of the system.

\section{RELATED WORK}

This project builds on previous work in educational technology in two main areas. The first area, computer graphics and animation, has a long history of engagement with education. Computer graphics have been used to teach chemistry by visualizing molecules (Wilson, 2002), astronomy through collaborative virtual reality (Hay et al., 2002), history through interactive history-based games (Jenkins, 2002) and many other topics. Animated characters are becoming common in education and training simulations, e.g., (Hill et al., 2003). Affective characters, in particular, are also being used to help the educational process (Schaub et al., 2003). The system presented here offers graphics that are competitive with the high end of modern computer games, using 3D autonomous characters, real-time shadows, and particle system fire and water effects.

Mobile computational devices are also on the rise in the popular cultures of industrialized societies, with the rapid penetration of cell phones and PDAs over the last few years. Numerous researchers have considered the capabilities of these mobile formats in educational contexts, e.g., (Roschelle \& Pea, 2002). The Teacher Education Program at MIT has done pioneering work in developing participatory simulations (Klopfer \& Woodruff, 2003). Other researchers have explored the use of mobile devices in education through a variety of different platforms (Borovoy et al., 1998). The project described in this paper uses mobile devices with high end graphics and built-in accelerometers (sensors for detecting the motion of the device); the use of these capabilities helps to create a novel mobile interaction with believable autonomous characters and virtual worlds, and distinguishes this work from previous research efforts.

This project also builds on previous work in autonomous characters and interactive installation design. The system extends a code base developed by the author and his collaborators, e.g., (Isla et al., 2001), and incorporates ideas in believable characters (Perlin \& Goldberg, 1996), affective computing (Picard, 1997) and biomorphic computation. By synthesizing elements from each of these areas, the project presented here offers a unique and novel educational interaction.

\section{INTERACTION PARADIGM}

A challenge in building virtual environments is deciding how real space and virtual space should relate to each other. An effective way to present the virtual/real boundary is to offer a metaphor that gives people a starting point for their understanding. For example, the metaphor in a flight simulator is that the user is a pilot sitting in the cockpit and the computer screen is the front windshield of the airplane. Other computational metaphors suggest that the computer screen is a "desktop" and that areas of the screen are "windows."

The central metaphor presented in this paper draws a parallel between the virtual space/real space distinction and the commonly understood distinction between land and water. This core "Land/Water" metaphor has served as the primary focus in developing all elements of the interaction paradigm and has had numerous implications for the design of the system. The core elements of the interaction paradigm are: animated creatures, virtual islands, virtual rafts and human participants. This section addresses each of these elements in turn.

The goal of this interaction paradigm is to provide a way for human participants to engage with animated creatures. These creatures provide the central focus for the educational content to be delivered by the system. Each stationary computer screen provides a fixed window onto an island community inhabited by the animated creatures. The screen itself serves as the boundary between virtual land - the area where the virtual creatures live and that people can see - and virtual water - the "gulf" of real space where people live and virtual creatures can not go. The animated creatures are constrained to exist on a virtual island, but are able to look out over the virtual water and interact with things they see there (by means of a web cam mounted on top of the screen). 
While the virtual islands allow people to have some simple interactions with the virtual creatures via the web cams on each island, the primary mode of interaction is through several Tablet PCs that people carry around the installation space. These Tablet PCs serve as virtual rafts that are able to carry creatures and interact with the islands and with each other. Each raft has a water simulation running on it, with simple real-time waves and an animated wooden platform in the middle of the screen (see Figure 3 ). The Tablet PCs have two-axis accelerometers built into them, which provide information about how the device is moving. These accelerometers make it possible for the character to react when the participant tilts the device. This simple mode of interaction, where the virtual raft responds to real world gravity, creates a strong link between the virtual and real worlds.

This interaction paradigm is designed to situate human participants at the conceptual center of the installation. People use

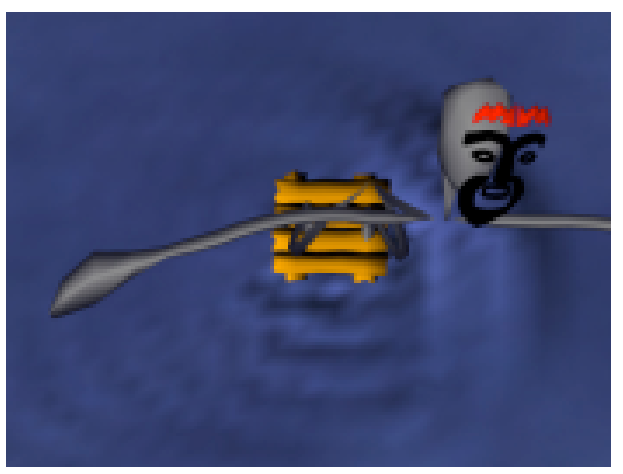

Figure 3: A view of the screen on the tablet PC based virtual raft. the rafts to help characters move onto new islands; in this regard, the participants serve the role of an ocean current or other force of nature. By putting people in a position to move creatures around the world and view the ensuing results, the project seeks to enable an active engagement with the ideas presented by each installation. This centrality of the participants will be particularly important in the upcoming ecology-based version of the project (see Future Work section below), which seeks in part to demonstrate that environmental stewardship is an active, hands-on process.

\section{PLATFORM FOR EDUCATION}

The virtual raft interaction paradigm has been designed to help people have interactive educational experiences. Storytelling, and in particular interactive storytelling, have been used effectively as learning tools (Rossiter, 2002; Roussos et al., 1997). The Virtual Raft paradigm provides many of the ingredients of good stories characters, voyages, encounters with different kinds of creatures - and thereby encourages people to find stories in the events that they find there. By giving participants the raw materials of stories, the project lets them to create their own narratives through which they can assemble an understanding of the material presented.

The paradigm also provides a fertile platform for providing a range of educational content. The current installation has been developed with humanoid characters as a means for addressing color theory, social content and mythology. Another version is currently bring planned in collaboration with the Discovery Science Center in Orange County, CA, that features animated versions of several animal species and may be used to help students learn ecological concepts such as the spread of biodiversity and the impact of invasive species.

The value of this paradigm comes not just from its ability to be adapted to a range of different content domains, but also from its ability to encourage people to engage in critical thinking. The system does not necessarily prescribe a certain set of ideas, but instead allows participants to interact with a system and discover for themselves the effects of their actions. While the computational design of each implementation will determine the cause-and-effect relationships to which participants will be exposed, the value judgments may be left to the participants themselves.

This paradigm also encourages participants to engage in real-time problem solving. Each version of the system has a multiplicity of outcomes based on how people interact. The design of the system allows people to try out certain solutions and then attempt to change the impact that they have had. While people's actions are effectively irreversible, there is nevertheless an abundance of opportunities to recover from previous actions. By combining the irreversibility of action and the potential to recover from mistakes with multiple user-defined opportunities for "success," the system encourages an active engagement with the concepts being presented.

\section{EVALUATION}

A fully functional prototype based on this paradigm was created in summer 2004. This prototype was exhibited at the opening of a new building on the campus of the University of California, Irvine, in November 2004. Approximately 200 participants interacted with the installation over two days, in nine groups of 20 and several small groups. These participants included industry professionals, academic administrators, professors, college students and a few grade school children. Each group visited the installation for a total of ten minutes. This visit consisted of a two minute introduction to the overall research, a one minute orientation to the installation itself, a five minute interaction with the installation, and two minutes for questions at the end. While it was not possible to collect data through questionnaires or other experimental instruments at this event, the video cited above offers some first hand evidence that the installation had the impacts described in this section.

The focus of this installation was to teach participants how the colors of light blend together. When each group of participants arrived, the three islands had red, green and blue fires on them, and each of the islands was inhabited by three characters with torches the same color as the fire. Whenever a character moved to a new island, it would walk over to the fire on that island and add its torch color to it. Therefore, if a participant 
carried a character with a red torch to a green island, the red character would walk over to the green fire and the fire would turn yellow. The participants were assigned the task of using the three rafts to disperse the characters so that each island ended up with white fire on it. To accomplish this task required at least six successful character transmissions (a character with a red torch needed to go to the blue island and the green island, a blue character needed to go to red and green islands, and a green character needed to go to the blue and red islands).

All nine large groups completed the task of turning all three fires white within the allotted five minutes. In the course of observing 200 people interact with the installation, several hypotheses about the system were drawn. First, the success of all nine groups supports the idea that the interaction paradigm draws on a metaphor that people readily understand. Since each group was able to perform the assigned task after only one minute of introduction to the interaction, the island/raft interface appears to be intuitive. People of all ages were willing to carry the virtual raft interface and convey characters around the space. While 200 people mostly from southern California does not represent a cross sample of humanity, it did appear that the installation did not rely upon a given language or cultural perspective for the core interaction paradigm to retain its effectiveness.

Second, since people took turns carrying the interface, the paradigm is transferable and lends itself to cooperation among multiple users. Whereas many mixed or augmented reality environments require some sort of tethered interface such as a heads up display or data glove, this interaction paradigm allows people to move fluidly in and out of the role of primary interactor without any significant changeover cost. The multi-user aspect of the virtual raft system caused the groups to work together to accomplish the task, including both raftholders and bystanders. It was common to hear exclamations such as, "Ooh, this island has a yellow fire, so it needs somebody with a blue torch!" and to have people share information with each other about where characters of various colors were in the space

A third feature of the system is that the interaction appears to be engaging to people. When each group, which had been through an hour of speeches and were now at the end of an hour-long tour of projects, reached the installation space, they were largely quiet and subdued. However, once the interaction with the Virtual Raft Project began, they became much more animated. During the five minute interaction, members of each of the groups talked and interacted physically with each other. At the end of several interactions, when the group accomplished its goal, the participants broke into spontaneous applause. This applause was not directed at the people demonstrating the project, but rather at the group itself for succeeding at its task.

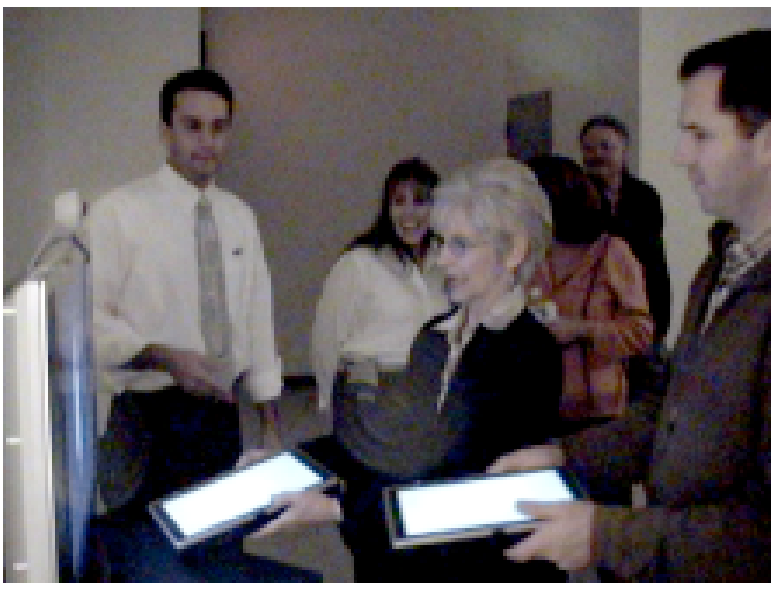

Figure 4: Multiple participants may interact with the installation simultaneously by means of several virtual rafts.

\section{FUTURE WORK}

This project is developing in two main directions. First, this system could be used as a platform for exploring and learning about social and cultural phenomena. Themes such as the spread of cultures, acceptance/discrimination, communication and teamwork could all be addressed by means of a system in which characters encounter communities that are new to them and try to find their place in that social system. In order to develop these facets of the system, it will be necessary to build a more comprehensive system of social intelligence in the characters. Previous work by the author has focused on social intelligence in virtual wolves (Tomlinson, 2002), and will provide a starting point for human-like computational social competence. In particular, mechanisms for characters to remember information about their world and to communicate this information to each other will be important parts of this future implementation.

The research team that created the Virtual Raft Project is also currently in the design stages for a version of the system based on ecology. By letting people move animated species among several virtual islands, the installations will allow them to understand concepts such as the spread or contraction of biodiversity, the role of predator/prey interactions, and the impact of invasive species. This project is being designed in consultation with educational curators at Discovery Science Center in Orange County, CA. The creators are developing it with an awareness of the California Science Standards (in particular focusing on the life sciences curriculum); the system will be evaluated based on its effectiveness at helping visitors learn that content. In order to be exhibited in a science center, it will be necessary to make the mobile elements of the system more robust to handling by thousands of children every year. In addition to producing a California specific installation, the system will be designed to produce a series of regionally specific installations featuring species from a range of regional ecosystems. By designing the system from its inception to develop multiple versions, the research team hopes to make it significantly easier to be able to develop new bodies of content for the same core interactive platform. The broad goal of the project is to give visitors to regional science centers and museums an 
opportunity to think critically about the science of ecology by engaging with locally relevant ecological themes in an interactive setting.

\section{CONCLUSIONS}

This paper has presented a novel interaction paradigm for multi-user participatory simulations. This paradigm involves desktop computer screens that serve as virtual islands populated by autonomous 3D animated creatures, and mobile devices, such as tablet PCs, that serve as virtual rafts that participants may use to carry creatures between the islands. This paper described how this "Island/Raft" paradigm may serve as an interactive platform for education in several different content domains, and presented a functional implementation of the paradigm.

This paper and the research it represents contribute to a new generation of educational systems that draw on technological advances in mobile computing, ubiquitous computing, computer graphics and artificial intelligence, and compete effectively against emerging forms of interactive entertainment. By harnessing these technologies and drawing elements from entertainment media to create an engaging experience, computational systems will be able to present content in ways that are of interest to learners and growing effectiveness as educational tools.

\section{ACKNOWLEDGEMENTS}

The author would like to thank Man Lok Yau, Jesse Gray, Sara Goetz, Eric Baumer, Jessica O’Connell, Ksatria Williams and So Yamaoka for their work on the Virtual Raft Project, as well as the staff of Discovery Science Center. This research was supported by the ACE (Arts Computation Engineering) program, the Donald Bren School of Information \& Computer Sciences, the Claire Trevor School of the Arts, the UC Game Culture \& Technology Lab, and the California Institute for Telecommunications \& Information Technology.

\section{REFERENCES}

Borovoy, R., Martin, F., Vemuri, S., Resnick, M., Silverman, B., \& Hancock, C. (1998). Meme tags and community mirrors: Moving from conferences to collaboration. Paper presented at the Proceedings of the 1998 ACM conference on Computer supported cooperative work.

Hay, K. E., Elliott, D. M., \& Kim, B. (2002). Collaborative network-based virtual reality: The past, the present, and the future of the virtual solar system. Paper presented at the Proceedings of the Computer Support for Collaborative Learning: Foundations for a CSCL Community.

Hill, R. W., Douglas, J., Gordon, A., Pighin, F. P., \& van Velsen, M. (2003). Guided conversations about leadership: Mentoring with movies and interactive characters. Paper presented at the Proceedings of the Fifteenth Innovative Applications of Artificial Intelligence Conference (IAAI-03), Acapulco, Mexico.

Isla, D., Burke, R., Downie, M., \& Blumberg, B. (2001). A layered brain architecture for synthetic creatures. Paper presented at the Proceedings of the International Joint Conferences on Artificial Intelligence (IJCAI), Seattle, WA, USA, August 4-10.

Jenkins, H. (2002). Game theory: Digital renaissance. MIT Technology Review, 1-3, March 29.

Klopfer, E., \& Woodruff, E. (2003). Platforms for participatory simulations - exploring systems and generating discourse with wearable and handheld computers. Paper presented at the Conference on Computer Supported Collaborative Learning, Oslo.

Perlin, K., \& Goldberg, A. (1996). Improv: A system for scripting interactive actors in virtual worlds. Paper presented at the Proceedings of ACM SIGGRAPH 96, New Orleans, LA, USA, August 4-9.

Picard, R. (1997). Affective computing. Cambridge, MA: MIT Press.

Roschelle, J., \& Pea, R. (2002). A walk on the wild side: How wireless handhelds may change cscl. Paper presented at the Proceedings of the 2002 Conference on Computer Supported Collaborative Learning, Boulder, CO.

Rossiter, M. (2002). Narrative and stories in adult teaching and learning. Clearinghouse on Adult, Career and Vocational Education, 241.

Roussos, M., Johnson, A. E., Leigh, J., Vasilakis, C. A., Barnes, C. R., \& Moher, T. G. (1997). Nice: Combining constructionism, narrative and collaboration in a virtual learning environment. SIGGRAPH Comput. Graph., 31(3), 62--63.

Schaub, H., Zoll, C., \& Aylett, R. (2003). Modelling empathy: The eu-project victec (virtual information and communication technology with empathic characters). Paper presented at the Fifth International Conference on Cognitive Modeling, Bamberg, Germany, April 10-12.

Tatar, D., Roschelle, J., Vahey, P., \& Penuel, W. R. (2003). Handhelds go to school: Lessons learned. Computer, 36(9), 30--37.

Tomlinson, W. (2002). Synthetic social relationships for computational entities. Unpublished Ph.D., Massachusetts Institute of Technology, Cambridge, MA.

Wilson, E. K. (2002). Computer learning hits its stride. Chemical \& Engineering News, 80, 35-39, 9/16/02. 